\title{
Human Weapon-Activity Recognition in Surveillance Videos Using Structural-RNN
}

\author{
Praneeth Susarla \\ University of Oulu \\ Oulu, Finland \\ praneeth.susarla@oulu.fi
}

\author{
Utkarsh Agrawal \\ IIITBangalore \\ Bangalore, India \\ utkarsh.agrawal@iiitb.org
}

\author{
Dinesh Babu Jayagopi \\ IIITBangalore \\ Bangalore, India \\ jdinesh@iiitb.ac.in
}

\begin{abstract}
In today's world, video surveillance systems play a vital role in commercial and industrial environments. The important goal of a surveillance activity is to observe suspicious behavior of humans and objects in a scene using camera or other sensors. Most of the current surveillance systems perform such activities by identifying persons, tracking their individual paths independently, not in conjunction with the objects in the scene. However, in a real world surveillance scenario,the behavior of people and their interaction with objects need to be modeled to reason about suspicious activities. Our contribution, through this work is in using the state-ofthe-art Structural Recurrent Neural Networks (SRNN) method to model the complex spatio-temporal human-object interactions in surveillance. Our best results have a final $F_{1}$ score of 87.3 on the human sub-activity recognition task and 82.7 on the object affordances recognition task. Our work considered weapons as objects of interest.
\end{abstract}

\section{CCS CONCEPTS}

- Human-centered computing $\rightarrow$ Activity centered design; • Computing methodologies $\rightarrow$ Activity recognition and understanding; Neural networks; Object detection; Video segmentation; Tracking;

\section{KEYWORDS}

Video Surveillance, Structural RNN, Tracking, Human-Object Interaction

\section{ACM Reference format:}

Praneeth Susarla, Utkarsh Agrawal, and Dinesh Babu Jayagopi. 2018. Human Weapon-Activity Recognition in Surveillance Videos Using Structural-RNN. In Proceedings of Mediterranean Conference on Pattern Recognition and Artificial Intelligence, Rabat, Morocco, March 27-28, 2018 (MedPRAI '18), 7 pages. https://doi.org/10.1145/3177148.3180080

\section{INTRODUCTION}

Automated video surveillance system $[28,30]$ is one of the important application areas of Computer Vision research. Recently, there

Permission to make digital or hard copies of all or part of this work for personal or classroom use is granted without fee provided that copies are not made or distributed for profit or commercial advantage and that copies bear this notice and the full citation on the first page. Copyrights for components of this work owned by others than ACM must be honored. Abstracting with credit is permitted. To copy otherwise, or republish to post on servers or to redistribute to lists, requires prior specific permission and/or a fee. Request permissions from permissions@acm.org.

MedPRAI '18, March 27-28, 2018, Rabat, Morocco

(C) 2018 Association for Computing Machinery.

ACM ISBN 978-1-4503-5290-1/18/03 ..\$15.00

https://doi.org/10.1145/3177148.3180080

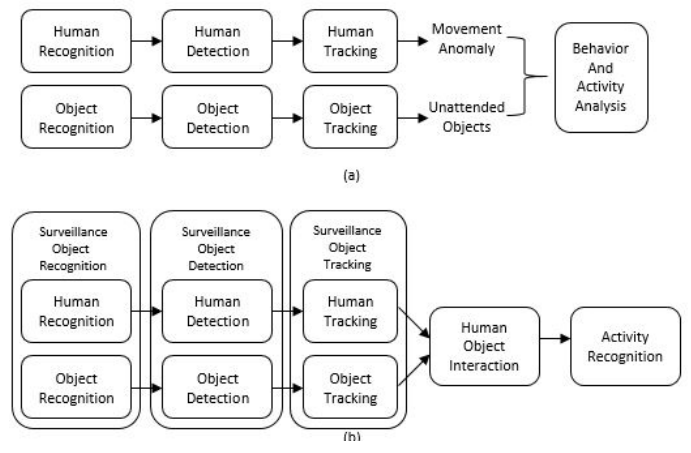

Figure 1: Pipeline of a video-surveillance system

has been frequent occurence of major terrorist attacks all over the world. In fact, according to IHS technology report ${ }^{1}$, global attacks have increased by nearly 27.5 percent from 2015-2016. Besides, these concerns have increased the usage of cameras for video surveillance. It is estimated that by 2015 , about $200,000,000$ cameras producing millions GB of data per week ${ }^{2}$ are in operation both at public and private places. This, has made it difficult even for human operators such as security guards, police officers etc. to quickly analyse and review the recorded videos resulting in inefficiencies due to manual surveillance[17]. Thus, there is an increase in need for automatic video-surveillance systems for better safety and security. The primary aim of these systems is to provide automatic interpretation of scenes and also predict the interactions between objects based on the information acquired by the sensors. The growth in this area is largely driven by the increased availability of inexpensive computing power and image sensors, as well as by the inefficiency of manual surveillance and monitoring system [30].

One of the major paradigms in current video surveillance systems is that they involve separate analysis of individual humans and objects using object detection [2, 8, 11, 19], tracking [2, 7, 8, 23], trajectory and human activity analysis [24, 27, 29]. Most of the surveillance systems created for commercial purposes tend to use a specific-purpose hardware along with an increased use of intelligent digital camera's ${ }^{3}$. The creation of a distributed automatic surveillance system by developing multi-camera or multi-sensor surveillance systems, fusion of information obtained across cameras $[4,5,15,25]$ especially to create an integrated system ${ }^{4}[21,25]$ are also active areas of this research.

\footnotetext{
${ }^{1}$ https://www.ihs.com/index.html

${ }^{2}$ IHS technology report, June 2015

${ }^{3} \mathrm{http}: / /$ www.detec.no, http://www.gotchanow.com

${ }^{4}$ http://dilnxsvr.king.ac.uk/cromatica/
} 
In our work, we consider a different pipeline for surveillance, as shown in Fig 1(b), by following a Human-Object Interaction (HOI) perspective. This pipeline is inspired from the semi-supervised video representation work proposed by Carlos et.al in [6]. We want to build a video-surveillance system with a common pre-trained recognizer, detector and a tracker for all the surveillance objects, so as to deal with all of them simultaneously by following a HOI approach. We aim at understanding the interactions among surveillance objects through their activities and sub-activities, provide more semantically meaningful and actionable knowledge over usual independent tracking of objects on RGB data. We use the current state-of-the-art Structural RNN technique, for automatically learning such interactions. So the paper has two contributions 1. Use of Structural RNN in surveillance type application. 2. The use of RGB data instead of RGB-D, which was used in the the original paper that proposed SRNN [13].

\section{STRUCTURAL RECURRENT NEURAL NETWORKS}

In real world, the interactions between objects and their surroundings are spatio-temporal in nature. Computer vision related problems like HOI frequently encounter spatio-temporal interactions. Such interactions in general are represented as spatio-temporal graphs and modeled using Conditional Random Fields (CRF) [16] or Bayesian Networks [22]. Structural Recurrent Neural Networks (SRNN) [13] is one such novel framework for modelling interactions using deep architectures. It is an approach proposed for combining the power of high-level spatio-temporal graphs and sequence learning success of Recurrent Neural Networks (RNN). The proposed method is generic and principled as it can be used for transforming any spatio-temporal graph (s-t graph) into a rich RNN mixture that is feedforward, fully differentiable and jointly trainable [13].

SRNN has shown its impact in spatio-temporal applications such as human motion modelling [13], human activity detection [13], anticipating driver maneuvering [12], 3d action recognition [18] etc. It has out-performed the state-of-the-art results in each one of these applications. It can model the real world spatio-temporal problems in a structured way (spatio-temporal manner) and yield superior results. Through our work, we use SRNN to model the surveillance problem by following a HOI approach and represent it as a spatio-temporal graph.

\section{SRNN FOR SURVEILLANCE}

The following are the reasons to consider learning interactions over simple trajectory tracking for surveillance activities in our work:

\subsection{Behaviour of the objects in a environment are highly correlated}

Behaviour of one object in a scene, can be dependant on other objects as well. For example, consider a case where a person approached a gun, lifted and began shooting in an environment. The weapon which was in stationary state, has changed its state to moving because of its interaction with the human. Similarly, the change of human state from walking to lifting followed by shooting occurred because of presence of weapon in the environment. Thus, the change in state of objects in the environment are correlated with one another. Learning the interactions between humans and weapons can help in identifying the states of multiple surveillance objects, all at the same time thereby, understanding the surveillance situation very effectively. It can help in analysing the sequence of events that occur in the surveillance environment in a structured manner.

\subsection{Importance of considering usability and scalability issues}

As most of the current surveillance systems [2, 5, 28] track each object independently and then analyse them accordingly, increase in the number of objects (either same or different type) of the environment increases the complexity of surveillance. If one has to accommodate this surveillance activity in real time, then this complexity can result in resource contention. However, if the same scenario is performed in a structural manner using neural networks [13], the surveillance task can still be run in real time with very minimal changes in the architecture during training of neural networks.

\subsection{Our Solution}

SRNN is one such method proposed by Jain et. al. [13], that can be helpful in solving the surveillance problem by learning their interactions. Factor sharing of SRNN method helps in dealing with varied number of objects in the surveillance environment. Such a SRNN can be used for automatically detecting suspicious activities such as 'lifting a gun' and 'pointing a gun', leading to better alerts and proactive responses. Also, most of the current videosurveillance systems need a human to constantly monitor them [17][28]. Instead, automating the detection of suspicious activities can enhance video-surveillance systems.

\section{INDIAN INDOOR SURVEILLANCE DATASET (IISD)}

As an initiative to prove that SRNN is highly useful in surveillance scenario, we have made our dataset similar to CAD-120 [14] but with RGB data. We have considered indoor surveillance as our application and created an RGB surveillance dataset (IISD). IISD contains 55 videos in total formed out of 5 ( 3 female, 2 male) different subjects. We identified 6 different high level human-weapon activities performed using 2 different weapon objects and also depicted 10 different sub-activties that are possible among them. The activities and sub-activities defined in for this dataset are inspired from complex activities and activities in [6]. We also defined 8 different weapon affordances in order to understand the weapon states independently during surveillance activities. Detailed specifications of IISD data are mentioned in Fig 2

As real weapons are not permitted, we have used toy weapons for AK-47 and revolver that resemble them, for our data collection. Some of the samples from the dataset are as shown in Fig 3

\subsection{Formation of $s-t$ graph for Surveillance}

The surveillance problem can be represented as an s-t graph as depicted in Fig 4. In IISD surveillance, human, AK-47 weapon and revolver weapon are the objects of our interest. We represent, AK-47 


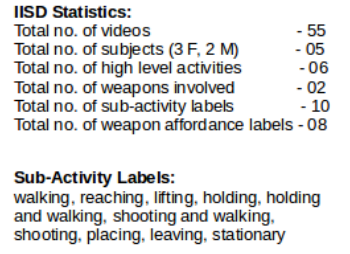

IISD High Level Activities:

in with gun, Dropping down the gun, Walking with the gun, Shooting with the gun,

Weapons: Ak-47, Revolver

Weapon Affordance labels: Stationary, moving, reachable, liftable, holding, shooting, placeable, leavable
Figure 2: IISD characteristics

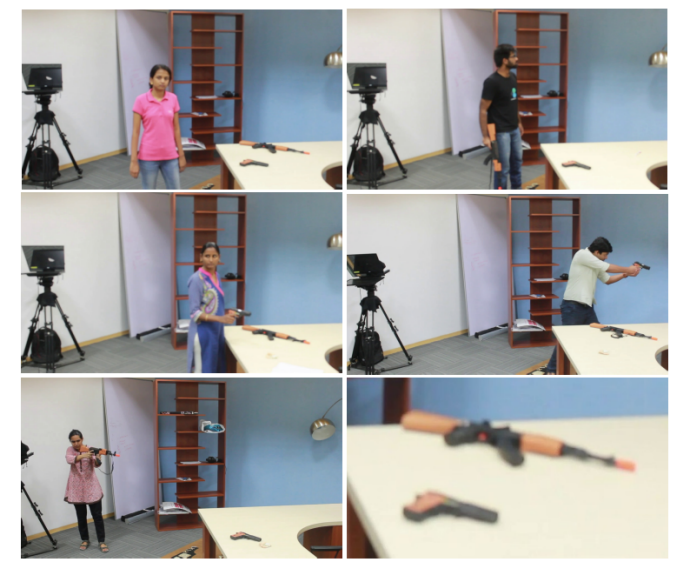

Figure 3: IISD sample images

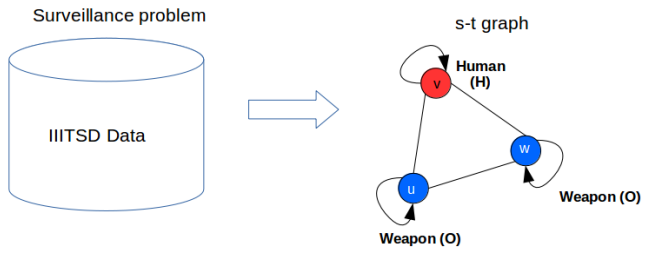

Figure 4: surveillance problem to s-t graph

and revolver under the same category "weapon" and deal with them accordingly in SRNN. Thus, there will be three nodes (human, AK-47 and revolver) of two different types (human, weapon) represented in an s-t graph as shown in Fig 4

\section{EXPERIMENTAL RESULTS}

\subsection{Preprocessing}

The IISD collected for surveillance is pre-processed with procedures such as bounding box annotations, joint position estimation for humans and temporal segmentation.

We annotated bounding boxes of surveillance objects in every IISD video frame in a semi-manual manner with the help of a Particle Filtering based tracker [31] and manual bounding box coordinates at select frames. Later, we automated this using deep learning based object detection methods. For joint position estimation, we used an open-source neural network model that estimates joint positions based on Iterative Error Feedback (IEF) mechanism [3]. The centroid of the human bounding box annotations in every frame is given as input body point to this method and joint estimations are captured. Temporal segmentation is one of the crucial pre-processing step, as its naive performance can follow up with poor human weapon-activity recognition. In our work, we manually segmented IISD data temporally.

\subsection{Feature Extraction for s-t graph}

Based on the s-t graph formation for IISD data representing surveillance, we divide all possible features in the graph into 6 different types. Table 1 provides a summary of all features for IISD s-t graph. The feature collection corresponding to Human and Weapon temporal in Table 1, have been modified in accordance to our surveillance problem. All other feature extraction templates are inspired from RGBD CAD-120 dataset [14].

In Table 1, human nodal features represent a vector of features computed from human joint location information using the IEF mechanism on RGB videos [3]. We compute the body pose (N1) feature templates mentioned in Table 1, using the joint orientation information of 10 upper skeleton joints (head, upper neck, right shoulder, left shoulder, right elbow, left elbow, right hand node, left hand node, thorax and pelvis) relative to torso position. Foot positions relative to pelvis joint location information are also computed to capture the foot movements with respect to upper body. One additional feature to capture sitting or standing position of the human is also considered by computing the clockwise angle between hip and head joint positions. Similary, we compute hand pose feature of the human (N2) using relative hand node positions relative to torso, maximum and minimum vertical hand positions within the temporal segment. Local features (N3) correspond to relative joint location information with respect to head position (neck, torso, left shoulder, right shoulder, left elbow, left hand node, right elbow and right hand node), distance and displacement for each one of these joints within the temporal segment. Thus, there are 69 different features captured N1, N2 and N3 templates. We then perform cumulative binning of all these features values into 10 bins. For further information about cumulative binning refer Appendix 7.2. We represent this as $\phi_{h}(i) \in \mathbb{R}^{690}$. For further details regarding these notations, refer factor graph parameterization in Fig 7

Weapon Nodal Features include object parameters, SIFT-matching features and local features. All these features together represent features of a weapon node in the graph within each temporal segment. Under Object parameters (N4), we compute bounding box and centroid location coordinates of the weapon node at the middle frame of the temporal segment. In N5 feature template, we compute the transformation matrix of SIFT matching points using SIFT descriptor [20] and Flann based matcher. This matching is computed between the weapon bouding box regions of middle frame and its previous frame within each temporal segment. In a similar manner, we compute weapon local features using their bounding box position information, distance and displacements of their centroid within a temporal segment, thus giving us $\phi_{o}(i) \in \mathbb{R}^{140}$ with $i$ representing a weapon node in s-t graph. 
Spatial features correspond to the features computed within a temporal segment, whereas temporal features capture the change across temporal segments. $\phi_{t}(i, j)$ represent the edge features formed by node $i$ and $j$ where $t$ corresponds to a spatial feature or temporal feature of the segment. As spatial features, we compute relative geometric features such as difference in weapon centroid positions, joint location positions, distance between them etc. at first, middle and last frames of the temporal segment. We then perform cumulative binning and obtain $\phi_{1}(i, j) \in \mathbb{R}^{150}$ for weapon-weapon and $\phi_{2}(i, j) \in \mathbb{R}^{400}$ for human-weapon respectively. As temporal features, we use vertical change in position and distance between corresponding weapon and joint locations, thus obtaining $\phi_{3}(i, j) \in \mathbb{R}^{40}$ and $\phi_{4}(i, j) \in \mathbb{R}^{160}$ respectively.

\subsection{Activity Recognition using SRNN}

As mentioned in Fig 2, walking, moving, reaching, stationary etc. are some of the sub-level activities of Patrolling with gun, Dropping down the gun, Handling the gun etc. high level activities in IISD data. The feature templates of all types (nodal, temporal and spatial) between human and weapon mentioned in section 5.2 are collected for each such segment of IISD data. For all the 454 segments of IISD data, we ended up with 853 feature files, some containing both nodal and spatial features while some others containing temporal features of the segment.

Fig 5 shows the SRNN architecture we modeled for an IISD surveillance s-t graph using the method proposed by Jain et. al. [13]. In this SRNN architecture, $R_{V_{i}}, i \in\{1,2\}$ and $R_{E_{i}}, i \in\{1,2,3,4\}$ represent the RNN's, handling all the corresponding object nodes and human-object edges of the graph respectively after applying factor sharing mechanism (Appendix 7.1). For nodeRNN's we use a single Long Short Term Memory Network (LSTM) [10] with 256 size (no. of layers in the network), followed by a uniform softmax classifier, whereas for edgeRNN's we use a single layer LSTM of size 128. We followed orthogonal initialization for all the LSTM's and used tanh and sigmoid as the activation functions for our SRNN architecture. The segment level 853 feature files collected for our IISD data serves as input to this SRNN architecture. At each time step, the human nodeRNN outputs one of the 10 sub-activity labels and weapon nodeRNN outputs one of the 8 affordance labels respectively, as defined in our IISD data.

We used the open source SRNN implementation for activity detection from [13], modified in accordance to our feature data and performed our human-weapon sub-activity recognition. We perform training and testing the model in a 5-fold cross-validation manner. We initially split the data into 5 folds with each fold containing all the segments related information of a particular subject of IISD data. We term this as subjectwise folding. Thus, 5 sets of training and testing datasets are formed with these 5 cross validation folds, serving as an input to SRNN.

\subsection{Results}

Human node in the graph is associated with subactivity labels of suspicious activities. Similary, weapon nodes of the s-t graph associate weapon affordance labels of IISD data. Some of the details of our SRNN architecture are mentioned in section 5.3. We perform this
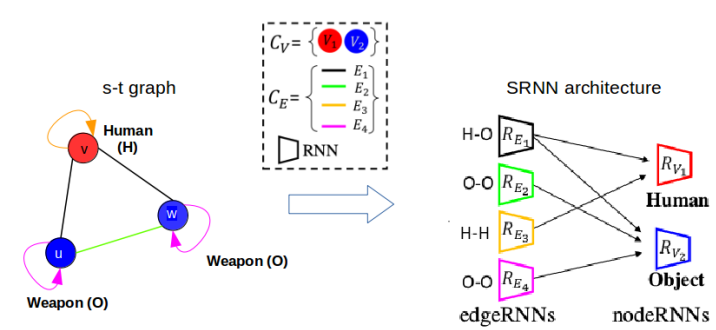

Figure 5: s-t graph to SRNN architecture for IISD data

human-weapon label prediction on SRNN architecture for recognition, anticipation and joint (both recognition and anticipation) tasks.

In recognition, the task of the network is to identify sub-activity of the current segment for human and affordance for both weapons (AK-47 and revolver) in test dataset. Similarly, in anticipation task, SRNN model predicts the label of next immediate segment in the test dataset. In joint recognition and anticipation task, model makes predictions about current segment as well as next consecutive segment labels in the test dataset. We use different labels each time on the same SRNN architecture(Fig 5), uniform softmax unit and cross-entropy loss function to perform each one of these tasks.

Through subjectwise folding, we train the SRNN network for 1000 iterations (no epochs are used) with the learning rate 0.001 in a cross-validation manner. We perform testing of the dataset at each cross-validation fold after every 10 iterations of the corresponding model training. The $F_{1}$ score results for each one of these, in all the 5 folds for both human and weapon activity recognition are mentioned in Table 2.

However, since the model training and testing is done in a crossvalidation manner, we calculate final $F_{1}$ scores for both human and weapon labels for the entire dataset by averaging out the $F_{1}$ score results of all the folds at their corresponding iterations and then compute the maximum of their averaged $F_{1}$ scores in all iterations. The final $F_{1}$ scores for human sub-activity recognition is 87.3 and object affordances recognition is 82.7

The current approach on IISD data is confined to semi-manual bounding box annotations and manual temporal segmentation during preprocessing. Some of these results on IISD video dataset are available at https://youtu.be/JbpUfvJBu1M and https://youtu.be/ IUBH40jkQFQ. Now, having witnessed promising results with this approach, we then made attempts to automate these pre-processing stages as they help in building a fully end-to-end automated surveillance system with this structured based approach.

As SRNN considers a segment level data as the input, automating bounding box annotations will be helpful during testing of the learnt model from this approach. To achieve this, we trained a common detector model for all the surveilance objects (human and weapons) using YOLO [26], a deep learning model based object detection. We even used KCF [9] and MIL [1] trackers along with detection on selected frames using the trained model with an attempt to reduce the overall running time for annotations. We chose run time complexities, number of bounding box annotations and accuracy 
Table 1: Summary of feature extraction for IISD s-t graph

\begin{tabular}{|l|l|}
\hline Feature Description & Count \\
\hline \hline Human Nodal Features & $\mathbf{6 9}$ \\
\hline N1: Body pose features & 25 \\
N2: Hand pose features & 12 \\
N3: Local features & 32 \\
\hline Weapon Nodal Features & $\mathbf{1 4}$ \\
\hline N4: Object parameters & 06 \\
N5: SIFT-method based features & 06 \\
N6: Local features & 02 \\
\hline Weapon-Weapon Spatial Features (computed & $\mathbf{1 5}$ \\
at start, middle and end frames, min and max) & \\
\hline E1: Difference in centroid locations & 02 \\
E2: Distance between centroids & 01 \\
\hline Human-Weapon Spatial Features (computed at) & $\mathbf{4 0}$ \\
start, middle, end frame, min and max) & \\
\hline E3: Distance between each joint location and object centroid & 08 \\
\hline Weapon Temporal Features & $\mathbf{0 4}$ \\
\hline E4: Vertical change in weapon bbox positions & 02 \\
E5: distance b/w corresponding weapon centroid locations & 02 \\
\hline Human Temporal Features & $\mathbf{1 6}$ \\
\hline E6: Vertical change in corresponding human joint positions & 08 \\
E7: Distance b/w correspoding human joint positions & 08 \\
\hline
\end{tabular}

Table 2: $F_{1}$ score results to only detection, only anticipation and joint detection with anticipation tasks, for both weapons and human object types in subjectwise folding on IISD data

\begin{tabular}{|c|c|c|c|c|c|c|c|c|}
\hline folds & \multicolumn{2}{|c|}{ only det } & \multicolumn{2}{|c|}{ only anti } & \multicolumn{2}{|c|}{ joint $\quad$ det } & \multicolumn{2}{|c|}{ joint anti } \\
\hline & $O$ & $H$ & $O$ & $H$ & $O$ & $H$ & $O$ & $H$ \\
\hline fold 1 & 87.7 & 95.9 & 63.7 & 65.4 & 91.6 & 87.8 & 67.3 & 64.3 \\
\hline fold_2 & 80.8 & 91.9 & 56.1 & 62.8 & 82.1 & 86.4 & 60 & 60.3 \\
\hline fold_3 & 84 & 86 & 64.4 & 62.8 & 88.6 & 82.8 & 68.9 & 66.8 \\
\hline fold_4 & 87.3 & 83.8 & 55.8 & 57.1 & 85.1 & 85.4 & 59.5 & 64.7 \\
\hline fold_5 & 91.8 & 97.9 & 63.1 & 71.3 & 82.1 & 94.6 & 65.4 & 75.0 \\
\hline Max_Avg & 82.7 & 87.3 & 55.2 & 57.8 & 82.2 & 85.5 & 58.6 & 60.9 \\
\hline
\end{tabular}

of the bounding boxes in terms of their Intersection Over Union (IOU) over the ground truth, as the parameters to evalaute the best possible method for automatic annotations in surveillance. Table 3, depicts the running time of various tracker with detection methods. Table 4 display the ratio of number of bounding box annotations obtained from the various methods to the number of groundtruth bounding box annotations for a $55 \mathrm{sec}$ video in IISD datatset. This ratio is less than 1 when method predicts less number of bounding box annotations and more than 1 when method predicts more number of annotations compared to their groundtruth annotations for a video. Similary, we even estimated the accuracies of bounding box annotations using classwise IOU as the measure between the predicted and groundtruth annotations. These estimated accuracies for various methods are enclosed in Table 5. For further details regarding these accuracy estimatoins refer Appendix 7.3. Based on the three chosen parameters, we found running the pre-trained detector on every frame, to be the best among all other methods for all videos in our IISD dataset.
We performed automatic temporal segmentation as well on the surveillance data using Aligned Cluster Analysis (ACA) [32] and Hierarchical Aligned Cluster Analysis (HACA) [33]. ACA and HACA are some of the widely used unsupervised methods for temporal segmentation. We modified the open source code put forth by Zhou et.al. [32, 33] for ACA and HACA accordingly and ran it on our RGB IISD dataset. Some of these results are shown in fig 6 . Our results are 80 percent accurate for temporal sequences with 2-4 clusters but got dropped to 50 percent, for sequences containing more than 4 sub-activities in our data. The reason for such performance could be because of insufficient features used for segmentation.It could also be due to the usage of a fixed temporal window length, for every sub-activity in the sequence in both ACA and HACA.

\section{CONCLUSION}

We achieved promising results on IISD data, proving the point that this method works in recognising suspicious activities under 


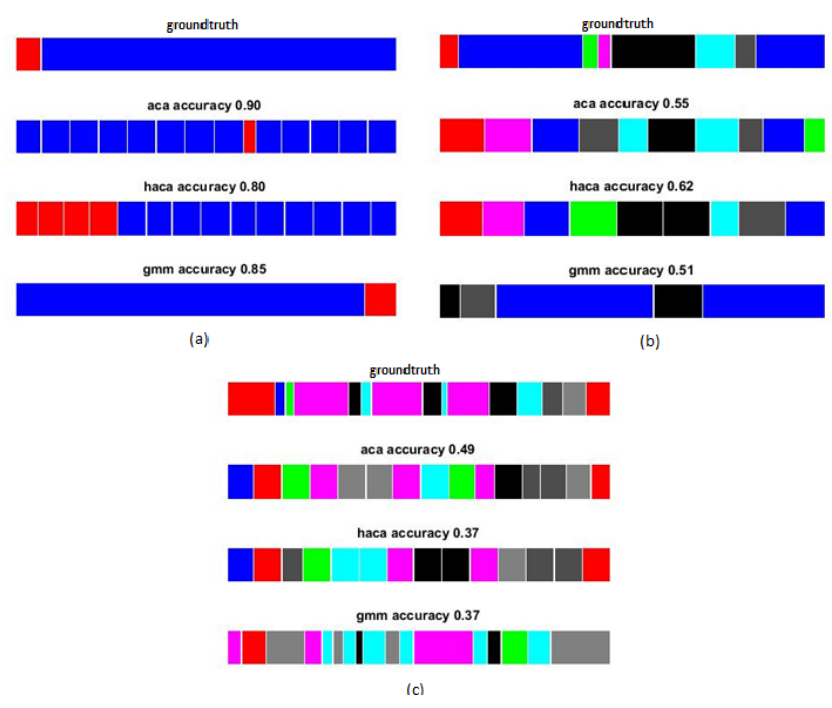

Figure 6: Temporal Segmentation results using ACA and HACA on IISD RGB data sequences with (a) 2 clusters for 2 different sub-activities (b) 5 clusters for 6 different subactivities and (c) 8 clusters for 9 different sub-activities respectively $[32,33]$

Table 3: Tracking and Detection results on a $55 \mathrm{sec}(1400$ frames approx.) recorded video from IISD dataset

\begin{tabular}{|l|l|}
\hline Method & Time(mm:ss) \\
\hline \hline Only detection & $01: 53$ \\
\hline Detection with KCF tracking over 5 frames & $04: 33$ \\
\hline Detection with KCF tracking over 10 frames & $05: 02$ \\
\hline Detection with KCF tracking over 15 frames & $05: 13$ \\
\hline Detection with MIL tracking over 5 frames & $04: 59$ \\
\hline Detection with MIL tracking over 10 frames & $05: 08$ \\
\hline Detection with MIL tracking over 15 frames & $05: 05$ \\
\hline
\end{tabular}

Table 4: Ratio of the number of model bounding box annotations to the ground truth bounding box annotations for a $55 s e c$ video (1400 frames approx.) in IISD dataset

\begin{tabular}{|l|c|}
\hline Method & Ratio \\
\hline \hline Only detection & 0.985 \\
\hline Detection with KCF tracking over 5 frames & 0.986 \\
\hline Detection with KCF tracking over 10 frames & 1.010 \\
\hline Detection with KCF tracking over 15 frames & 0.993 \\
\hline Detection with MIL tracking over 5 frames & 0.986 \\
\hline Detection with MIL tracking over 10 frames & 0.986 \\
\hline Detection with MIL tracking over 15 frames & 0.993 \\
\hline
\end{tabular}

surveillance. However, compared to IISD problem, the real surveillance scenario is complex involving more different kinds objects and multiple interactions between them. Through this work, we
Table 5: Accuracies of predicted bounding box annotations over their ground truth using IOU measure for a $55 \mathrm{sec}$ video in IISD dataset

\begin{tabular}{|l|l|}
\hline Method & Accuracy (\%) \\
\hline \hline Only detection & 94.597 \\
\hline Detection with KCF tracking over 5 frames & 94.486 \\
\hline Detection with KCF tracking over 10 frames & 92.249 \\
\hline Detection with KCF tracking over 15 frames & 93.106 \\
\hline Detection with MIL tracking over 5 frames & 94.436 \\
\hline Detection with MIL tracking over 10 frames & 92.765 \\
\hline Detection with MIL tracking over 15 frames & 93.699 \\
\hline
\end{tabular}

claim that this structured approach can be very effective in understanding such interactions automatically in real-surveillance situations.

The automatic bounding box annotation results as shown in fig 3 along with human weapon-activity recognition accuracies using SRNN provides scope to fully automate the entire process under surveillance. However, the current temporal segmentation methods like ACA and HACA (as shown in fig 6) are not adequate to perform reasonably accurate automatic surveillance activity recognition. Hence, our future work is on building an automatic temporal segmentation method using deep learning methods thereby, achieving a fully end-to-end automated activity recognition surveillance system using this structured approach.

\section{APPENDIX}

\subsection{Factor Sharing}

A factor graph is a type of probabilistic graphical model having two types of nodes namely Variables and Factors. Variables are nodes of the graph with their values either known or to be predicted. Factors on the other hand, define the relationship between variables in the graph. Each factor can be connected to many variables and comes with a factor function to define the relationship between these variables.

While converting a s-t graph into SRNN, we unroll the s-t graph in time and convert into a factor graph. This graph representation has a factor function $\Psi_{v}\left(y_{v}, x_{v}\right)$ for each node and a pairwise factor $\Psi_{e}\left(y_{e(1)}, y_{e(2)}, x_{e}\right)$ for each edge where $v$ and $e$ represent the vertex and edge of a s-t graph as shown in figure7. Each factor in the s-t graph has parameters that needs to be learned. Instead of learning a distinct factor for each node, semantically similar nodes can optionally share factors. For example, all object nodes u,w in the st-graph can share the same node factor and parameters. This modeling choice allows enforcing parameter sharing between similar nodes. It further gives the flexibility to handle st-graphs with more nodes without increasing the number of parameters [13].

\subsection{Cumulative Binning}

Given an arbitrary number of bins and a set of feature values, we initially define range for each bin and then identify the bin range suitable for each one of these feature values and then associate each of these feature values to the corresponding bins. This is the usual process of binning feature values. In cumulative binning, feature 


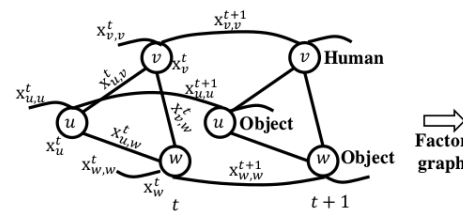

(b) Unrolled through time

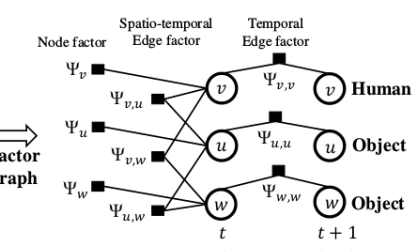

(c) Factor graph parameterization
Figure 7: Unrolled s-t graph to factor graph conversion [13]

values of a particular bin are also associated to all other upcoming bins. Thus, the binning of feature values is done in a cumulative manner. This process of binning features, can be useful in creating less sparse but higher dimensional features.

\subsection{Evaluation of IOU measure for Bounding Box Annotations}

In our work, we consider classwise data of predicted and ground truth bounding box annotations as the input for this measure. At first through IOU, we measure the ratio of area of overlap to the area of union of classwise predicted and ground truth bounding box annotations. If the IOU is greater than 0.5 , we consider it to be a good prediction. We then measure the accuracy of the method by calculating the ratio of total number of good predictions to the total number of overall predictions occurred through that method.

\section{REFERENCES}

[1] Boris Babenko, Ming-Hsuan Yang, and Serge Belongie. 2009. Visual tracking with online multiple instance learning. In Computer Vision and Pattern Recognition, 2009. CVPR 2009. IEEE Conference on. IEEE, 983-990.

[2] Terrance E Boult, Ross J Micheals, Xiang Gao, and Michael Eckmann. 2001. Into the woods: Visual surveillance of noncooperative and camouflaged targets in complex outdoor settings. Proc. IEEE 89, 10 (2001), 1382-1402.

[3] Joao Carreira, Pulkit Agrawal, Katerina Fragkiadaki, and Jitendra Malik. 2016 Human pose estimation with iterative error feedback. In Proceedings of the IEEE Conference on Computer Vision and Pattern Recognition. 4733-4742.

[4] Robert T Collins, Alan J Lipton, Hironobu Fujiyoshi, and Takeo Kanade. 2001 Algorithms for cooperative multisensor surveillance. Proc. IEEE 89, 10 (2001), $1456-1477$.

[5] Robert T Collins, Alan J Lipton, Takeo Kanade, Hironobu Fujiyoshi, David Duggins, Yanghai Tsin, David Tolliver, Nobuyoshi Enomoto, Osamu Hasegawa, Peter Burt, et al. 2000. A system for video surveillance and monitoring. VSAM final report (2000), 1-68.

[6] Carlos Fernando Crispim-Junior, Michal Koperski, Serhan Cosar, and Francois Bremond. 2016. Semi-supervised understanding of complex activities from temporal concepts. In 13th International Conference on Advanced Video and Signal-Based Surveillance.

[7] T Darrell, G Gordon, J Woodfill, H Baker, and M Harville. 1998. Robust, real-time people tracking in open environments using integrated stereo, color, and face detection. In Visual Surveillance, 1998. Proceedings., 1998 IEEE Workshop on. IEEE, 26-32.

[8] Ismail Haritaoglu, David Harwood, and Larry S Davis. 1999. Hydra: Multiple people detection and tracking using silhouettes. In Visual Surveillance, 1999. Second IEEE Workshop on,(VS'99). IEEE, 6-13.

[9] João F Henriques, Rui Caseiro, Pedro Martins, and Jorge Batista. 2015. High-speed tracking with kernelized correlation filters. IEEE Transactions on Pattern Analysis and Machine Intelligence 37, 3 (2015), 583-596.

[10] Sepp Hochreiter and Jürgen Schmidhuber. 1997. Long short-term memory. Neural computation 9, 8 (1997), 1735-1780.

[11] Yuri Ivanov, Aaron Bobick, and John Liu. 2000. Fast lighting independent background subtraction. International fournal of Computer Vision 37, 2 (2000), 199-207.

[12] Ashesh Jain, Hema S Koppula, Bharad Raghavan, Shane Soh, and Ashutosh Saxena. 2015. Car that knows before you do: Anticipating maneuvers via learning temporal driving models. In Proceedings of the IEEE International Conference on Computer Vision. 3182-3190.
[13] Ashesh Jain, Amir R Zamir, Silvio Savarese, and Ashutosh Saxena. 2016. Structural-RNN: Deep learning on spatio-temporal graphs. In Proceedings of the IEEE Conference on Computer Vision and Pattern Recognition. 5308-5317.

[14] Hema Swetha Koppula, Rudhir Gupta, and Ashutosh Saxena. 2013. Learning human activities and object affordances from $\mathrm{rgb}-\mathrm{d}$ videos. The International fournal of Robotics Research 32, 8 (2013), 951-970.

[15] John Krumm, Steve Harris, Brian Meyers, Barry Brumitt, Michael Hale, and Steve Shafer. 2000. Multi-camera multi-person tracking for easyliving. In Visual Surveillance, 2000. Proceedings. Third IEEE International Workshop on. IEEE, 3-10.

[16] John Lafferty, Andrew McCallum, and Fernando CN Pereira. 2001. Conditional random fields: Probabilistic models for segmenting and labeling sequence data. (2001).

[17] Po Kong Lai, Marc Décombas, Kelvin Moutet, and Robert Laganière. 2016. Video summarization of surveillance cameras. In Advanced Video and Signal Based Surveillance (AVSS), 2016 13th IEEE International Conference on. IEEE, 286-294.

[18] Jun Liu, Amir Shahroudy, Dong Xu, and Gang Wang. 2016. Spatio-temporal LSTM with trust gates for 3D human action recognition. In European Conference on Computer Vision. Springer, 816-833.

[19] Li-Chang Liu, Jong-Chih Chien, HY-H Chuang, and Ching-Chung Li. 2003. A frame-level FSBM motion estimation architecture with large search range. In Advanced Video and Signal Based Surveillance, 2003. Proceedings. IEEE Conference on. IEEE, 327-333.

[20] David G Lowe. 2004. Distinctive image features from scale-invariant keypoints. International journal of computer vision 60, 2 (2004), 91-110.

[21] Dimitrios Makris, Tim Ellis, and James Black. 2004. Bridging the gaps between cameras. In Computer Vision and Pattern Recognition, 2004. CVPR 2004. Proceedings of the 2004 IEEE Computer Society Conference on, Vol. 2. IEEE, II-II.

[22] Kevin P Murphy. 2012. Machine learning: a probabilistic perspective. MIT press.

[23] James Orwell, Paolo Remagnino, and GA Jones. 1999. Multi-camera colour tracking. In Visual Surveillance, 1999. Second IEEE Workshop on,(VS'99). IEEE, $14-21$.

[24] Jonathan Owens and Andrew Hunter. 2000. Application of the self-organising map to trajectory classification. In Visual Surveillance, 2000. Proceedings. Third IEEE International Workshop on. IEEE, 77-83.

[25] B Ping Lai Lao, Sun and S Velastin. 2003. Fusing visual and audio information in a distributed intelligent surveillance system for public transport systems. (2003).

[26] Joseph Redmon, Santosh Divvala, Ross Girshick, and Ali Farhadi. 2016. You only look once: Unified, real-time object detection. In Proceedings of the IEEE Conference on Computer Vision and Pattern Recognition. 779-788.

[27] Nathanael Rota and Monique Thonnat. 2000. Video sequence interpretation for visual surveillance. In Visual Surveillance, 2000. Proceedings. Third IEEE International Workshop on. IEEE, 59-68.

[28] Mubarak Shah, Omar Javed, and Khurram Shafique. 2007. Automated visual surveillance in realistic scenarios. IEEE MultiMedia 14, 1 (2007).

[29] Chris Stauffer and W. Eric L. Grimson. 2000. Learning patterns of activity using real-time tracking. IEEE Transactions on pattern analysis and machine intelligence 22,8 (2000), 747-757.

[30] Maria Valera and Sergio A Velastin. 2005. Intelligent distributed surveillance systems: a review. IEE Proceedings-Vision, Image and Signal Processing 152, 2 (2005), 192-204.

[31] Yunji Zhao and Hailong Pei. 2012. Model update particle filter for multiple objects detection and tracking. International fournal of Computational Intelligence Systems 5, 5 (2012), 964-974.

[32] Feng Zhou, Fernando De la Torre, and Jessica K Hodgins. 2008. Aligned cluster analysis for temporal segmentation of human motion. In Automatic Face \& Gesture Recognition, 2008. FG'08. 8th IEEE International Conference on. IEEE, 1-7.

[33] Feng Zhou, Fernando De la Torre, and Jessica K Hodgins. 2013. Hierarchical aligned cluster analysis for temporal clustering of human motion. IEEE Transactions on Pattern Analysis and Machine Intelligence 35, 3 (2013), 582-596. 